\title{
Forecast of Maize Production in Henan Province
}

\author{
Bingjun Li, Xiaoxiao Zhu* \\ College of Information and Management Science, Henan Agricultural University, Zhengzhou, China \\ Email: zzlbjun@163.com, ${ }^{\star}$ zhu_xiaoxiao113@163.com
}

How to cite this paper: Li, B.J. and Zhu, X.X. (2018) Forecast of Maize Production in Henan Province. American Journal of Plant Sciences, 9, 2276-2286.

https://doi.org/10.4236/ajps.2018.911164

Received: September 13, 2018

Accepted: October 26, 2018

Published: October 29, 2018

Copyright (C) 2018 by authors and Scientific Research Publishing Inc. This work is licensed under the Creative Commons Attribution International License (CC BY 4.0).

http://creativecommons.org/licenses/by/4.0/

\begin{abstract}
Considering the influence of meteorological factors on maize production, in order to improve the yield of maize in Henan Province, a grey combination model is constructed to predict the yield of maize in Henan Province. Firstly, the yield of maize in 2017 is obtained by GM $(1,1)$ model; secondly, the trend yield of maize is obtained by HP filter method, then the meteorological yield of maize is obtained, and the yield of maize reduction is determined according to the meteorological yield. Combined with Markov model, the maize yield reduction in various cities in Henan Province is forecasted. Finally, based on the reduction of production, policy recommendations are made for maize production in Henan Province.
\end{abstract}

\section{Keywords}

Grey System Theory, GM $(1,1)$ Model, Markov Model, HP Filter Method, Maize

\section{Introduction}

Henan Province is located in the middle and lower reaches of the Yellow River and the upper reaches of the Huaihe River. The land area is 167,000 square kilometers, accounting for 17.3 percent of the country's total area. Henan is a major grain producing Province, the stability of food production is related to people's basic needs. There are many factors affecting grain production: agricultural production technology, meteorological factors and policy price factors. Among them, as one of the main factors affecting grain production, the amount of meteorological factors investment is not controlled by humans. For meteorological disasters, all that people can do is to predict and prevent them in order to reduce their impact on grain production. The per unit area yield of maize in Henan Province was $5638 \mathrm{~kg} / \mathrm{hm}^{2}$ in 2012 and fell to $5285 \mathrm{~kg} / \mathrm{hm}^{2}$ in 2016 [1]. 
With the development of science and technology, the effect of agricultural production and policy price on maize yield is positive. Therefore, meteorological factors are the main factors that affect grain yield reduction.

The following scholars used different methods to study maize production, including: Ma Shuqing et al. [2] analyzed the correlation between maize seedling condition and maize yield, and provided a variety of evaluation models to evaluate the yield reduction of maize in spring drought. Sun Yulian et al. [3] explored the key meteorological factors affecting the growth of Linxia maize in Gansu Province and constructed a spatial-temporal dynamic climate prediction model for forecasting maize in different growth stages. Luo Zhengyuan et al. [4] used support vector machine (SVM) algorithm to predict maize yield in Heilongjiang Province. Zhang Wenying et al. [5] Li Guoqiang et al. [6] used grey GM $(1,1)$ model to predict maize yield in Hebei and Huanghuaihai. Jiang Hewen et al. [7] used a method of periodic correction of residual values to construct a grey prediction model to predict grain yield in Liaoning Province. Fang Huimin et al. [8] used BP neural network to predict the regional test yield. Su Hengqiang et al. [9] used the entropy weight method to determine the weight of a single model, and used combined forecasting model to predict maize yield. Hou Yingyu, et al. [10] established a dynamic forecasting model to predict the yield of maize and winter wheat in the United States. Wei Jie et al. [11] used exponential smoothing method to predict maize yield in Shanxi Province. Ma Jianrui et al. [12] used water-fertilizer coupling model to predict maize yield.

In addition, some scholars have studied the relationship between meteorological change and grain production. Wang Futang [13] used three atmospheric circulation models to predict possible changes in rice, wheat and maize production in China's major crops in future climate scenarios. Using the WOFOST crop model and the future climate scenario data from the climate model BCC-T63, Zhang Jianping et al. [14] simulated and analyzed the effect of future climate change on maize yield in Northeast China. Jin Zhiqing et al. [15] and Xiong Wei [16] used CERES-Maize to simulate the impact of global climate change on maize production and agricultural production in ecologically fragile areas in China. Chen Shang et al. [17] combined historical meteorological data, K-NN algorithm and CERES-Maize model to construct a dynamic prediction method for maize growth period. Shang Zongbo [18] used the maize growth physiological and ecological simulation model (MPESM) to analyze the growth trend and yield variation of spring maize in Shenyang under the background of global climate change. Jiqi [19] combined multiple regression forecasting model with disaster reduction rate to predict maize yield and make the forecast of yield tend to be quantitative.

The above scholars predict maize yield single, or establish crop model to predict maize yield. Less prediction was made on maize yield reduction. The grey GM $(1,1)$ model works well for short-term prediction. The HP filter method is reasonable for separating crop trend yield. In addition, the Markov model is suitable for the prediction problem of data series with large random fluctuations. 
Therefore, after using the GM $(1,1)$ model to obtain the corn yield in Henan Province in 2017, the HP filter method is used to obtain the trend yield of maize, and then the meteorological yield of maize is obtained. Finally, markov model is used to predict the yield of maize in Henan Province. And we provided policy recommendations for future maize production in Henan Province.

\section{Basis of Theory and Method}

In 1982, Professor Deng Julong published “The Control Problems of Grey systems" [20], which marked the birth of the emerging discipline of the grey system theory [21]. After more than 30 years of development, the grey system theory has been applied to the fields of agriculture, economy, society and industry, solved many problems in production and life.

\subsection{Grey GM $(1,1)$ Model}

Grey GM $(1,1)$ model is mainly used in the prediction of grey system theory in prediction. Grey GM $(1,1)$ model requires less data, can be tested, and has a high precision in short-term prediction. It has been widely used in many scientific fields such as industry, agriculture, energy, transportation, geology, meteorology, hydrology, ecology, environment, medicine, military, economy, society and so on, and has successfully solved a large number of practical problems in production, life and scientific research. The modeling mechanism is as follows:

Let an original sequence be:

$$
X^{(0)}=\left\{x^{(0)}(1), x^{(0)}(2), \cdots, x^{(0)}(n)\right\}
$$

Of which: $x^{(0)}(k) \geq 0, k=1,2, \cdots, n$.

Perform an accumulation (1-AGO) generation on the sequence to get the sequence:

$$
X^{(1)}=\left\{x^{(1)}(1), x^{(1)}(2), \cdots, x^{(1)}(n)\right\}
$$

$X^{(1)}$ adjacent mean generating sequence is:

$$
Z^{(1)}=\left\{z^{(1)}(2), z^{(1)}(3), \cdots, z^{(1)}(n)\right\}
$$

Of which $z^{(1)}(k)=\frac{1}{2}\left(x^{(1)}(k)+x^{(1)}(k-1)\right)$,We call

$$
x^{(0)}(k)+a z^{(1)}(k)=b
$$

the mean form of the GM $(1,1)$ model.

Let the model's parameter list be $\hat{a}=(a, b)^{\mathrm{T}}$, then use the least squares method to find

$$
\hat{a}=\left(B^{\mathrm{T}} B\right)^{-1} B^{\mathrm{T}} Y
$$

$Y, B$ of them are,

$$
Y=\left[\begin{array}{c}
y^{(0)}(2) \\
y^{(0)}(3) \\
\vdots \\
y^{(0)}(n)
\end{array}\right], B=\left[\begin{array}{cc}
-z^{(1)}(2) & 1 \\
-z^{(1)}(3) & 1 \\
\vdots & \vdots \\
-z^{(1)}(n) & 1
\end{array}\right]
$$


We call

$$
\frac{\mathrm{d} x^{(1)}}{\mathrm{d} t}+a x^{(1)}=b
$$

the whitening differential equation of $x^{(0)}(k)+a z^{(1)}(k)=b$.

Then the solution of Equation (6) is

$$
\hat{x}^{(1)}(t)=\left(x^{(0)}(1)-\frac{b}{a}\right) \mathrm{e}^{-a(t-1)}+\frac{b}{a}
$$

Then the simulated prediction difference form of the original sequence is

$$
\hat{x}^{(0)}(k)=\hat{x}^{(1)}(k)-\hat{x}^{(1)}(k-1)=\left(1-e^{a}\right)\left(x^{(0)}(1)-\frac{b}{a}\right) \mathrm{e}^{-a(k-1)}
$$

In this model, $a$ is the development coefficient and $b$ is the grey effect.

\subsection{Markov Model}

The research object of Markov Probability Matrix Prediction model is a stochastic dynamic system. Markov model is effective in predicting stochastic volatility problems. It predicts the development of the system according to the transition probability between states. The transition probability reflects the degree of influence of each random factor and the inherent regularity of each state. Markov Probability Matrix is suitable for predicting data columns with large random fluctuations [22].

The Markov forecasting model [23] is as follows:

Let $P_{i j}^{(k)}=P\left(X_{m+k}=j \mid X_{m}=i\right),(i, j) \in I$, which denote the probability that the system is in the state $j$ at time $m+k$ under the condition that the system in the state $i$ of at the moment $m$. That is, the transition probability of the $k$ step is experienced; by sequentially sorting $P_{i j}^{(k)}$, the following matrix can be obtained.

$$
P^{(k)}=\left[\begin{array}{cccc}
P_{11}^{(k)} & P_{12}^{(k)} & \cdots & P_{1 n}^{(k)} \\
P_{21}^{(k)} & P_{22}^{(k)} & \cdots & P_{2 n}^{(k)} \\
\vdots & \vdots & & \vdots \\
P_{n 1}^{(k)} & P_{n 2}^{(k)} & \cdots & P_{n n}^{(k)}
\end{array}\right]
$$

Then the matrix is the $k$-step transition probability matrix of Markov chain. Of which: $\sum_{j=1}^{n} P_{i j}^{(k)}=1$.

If $M_{i j}(k)$ is the number of original data samples transferred from state $\otimes_{i}$ to state $\otimes_{j}$ through $k$ steps, and $M_{i}$ is the number of original data samples in state $\otimes_{i}$, then call

$$
P_{i j}^{(k)}=\frac{M_{i j}(k)}{M_{i}}(i, j=1,2, \cdots, n)
$$

the state transition probability. Since the final state of the data sequence is not clear, the last $k$ data in the data sequence should be removed when calculating $M_{i}$. 


\subsection{HP Filter Method}

The HP filtering method is a decomposition method of time series in state space. It assumes that the time series consists of two parts, namely: long-term trend component and short-term fluctuation component. The HP filter method can be considered as a high-pass filter that can separate high-frequency components with periods below 8a [24].

Maize yield sequence $\left\{y_{t}\right\}$ is divided into trend yield $g_{t}$ and fluctuating yield $c_{t}$ by HP filter, in which $g_{t}$ is a stable high frequency trend signal and $c_{t}$ is an unstable low frequency disturbance signal. The principle is to remove the low frequency signal $c_{t}$ by filtering, thereby detecting the high frequency signal $g_{t}$ from the production sequence. HP filter decomposes $y_{t}$ into: $y_{t}=g_{t}+c_{t}$.

The detection method of high frequency signal $g_{t}$ is often defined as the solution of the following minimization formula, namely:

$$
\min \left\{\sum_{t=1}^{T}\left(y_{t}-g_{t}\right)+\lambda \sum_{t=1}^{T}\left[\left(g_{t+1}-g_{t}\right)-\left(g_{t}-g_{t-1}\right)\right]^{2}\right\}
$$

In the formula, $\lambda$ is the HP filter parameter. When $\lambda=0$, the minimized solution of the function is $\left\{y_{t}\right\}$ sequence. With the increase of $\lambda$ value, the trend of minimum solution estimation is smoother. The general experience of $\lambda$ is as follows:

$$
\lambda= \begin{cases}100, & \text { annual data } \\ 1600, & \text { quarterly data } \\ 14400, & \text { monthly data }\end{cases}
$$

\subsection{Calculation of Meteorological Production}

Crop yield is usually decomposed into three parts: trend yield, meteorological yield and random error. The trend output reflects the long-period production component of the level of productivity development in the historical period, also known as technical output. Meteorological production is a volatility that is affected by short-period changes in meteorological factors [25]. Trend yields often use various linear or nonlinear simulations with "time" as the independent variable. The general formula can be expressed as follows:

$$
Y=Y_{w}+Y_{t}+e
$$

where $Y$ represents the actual yield of maize, $Y_{t}$ is the trend yield, which represents the contribution of social and economic conditions and technological level of productivity to grain production. $Y_{w}$ is meteorological production, which characterizes the contribution of climate fluctuations to actual production. $\mathrm{e}$ is the component of yield affected by some random factors, which accounts for a small proportion and is often neglected in practical calculation. In general, the level of agricultural technology is gradually improved, so trend yield is usually a function of time, and its influence on yield is a more smooth process in time series, especially in a larger geographical area [26]. In this paper, the HP filter me- 
thod is used to obtain the trend yield of each year's maize, and then the meteorological yield of each year is calculated by $Y_{w}=Y-Y_{t}$.

\section{Data Collection and Collation}

The yield data set of Henan maize in 2006-2016 comes from Henan Statistics Bureau [1]. The research objects selected in this paper are 18 cities in Henan Province. In addition, the yield data of Henan Province in 2017 are obtained by GM $(1,1)$ model based on the data of the previous 10 years. The detailed data are shown in Table 1.

The trend yield of maize in 18 cities of Henan Province from 2006 to 2016 is obtained by using HP filter method. The calculated results are shown in Table 2.

For the year when the meteorological yield is less than 0 , we set the year of production reduction. For the year when the meteorological yield is greater than 0 , we set the year of production increase. Therefore, according to the Markov prediction model, the reduction of yield in various places can be predicted. Take Zhengzhou City as an example, set a reduction of production to $M_{1}$, and increase production to $M_{2}$, therefore

$$
M_{11}(1)=2, M_{12}(1)=4, M_{21}(1)=4, M_{22}(1)=1
$$

The state transition probability is

$$
p_{11}^{(1)}=\frac{1}{3}, p_{12}^{(1)}=\frac{2}{3}, p_{21}^{(1)}=\frac{4}{5}, p_{22}^{(1)}=\frac{1}{5}
$$

\begin{tabular}{|c|c|c|c|c|c|c|c|c|c|c|c|c|}
\hline & 2006 & 2007 & 2008 & 2009 & 2010 & 2011 & 2012 & 2013 & 2014 & 2015 & 2016 & 2017 \\
\hline Zhengzhou & 4940.38 & 4985.98 & 4870.00 & 4908.00 & 4828.00 & 4727.00 & 4818.00 & 4758.00 & 4562.79 & 4788.92 & 4593.14 & 4588.31 \\
\hline Kaifeng & 5459.22 & 5595.66 & 5505.00 & 5432.00 & 5866.00 & 5372.00 & 5465.00 & 5511.00 & 5359.33 & 5498.72 & 5246.42 & 5336.66 \\
\hline Luoyang & 4960.82 & 5210.71 & 4990.00 & 4943.00 & 4407.00 & 4652.00 & 4814.00 & 4755.00 & 4173.38 & 4796.20 & 4565.16 & 4405.71 \\
\hline Pingdingshan & 4722.73 & 4917.77 & 5062.00 & 4746.00 & 4738.00 & 4689.00 & 4872.00 & 4839.00 & 3369.38 & 4801.78 & 4617.68 & 4308.35 \\
\hline Anyang & 6140.49 & 6596.96 & 6682.00 & 6564.00 & 6461.00 & 6383.00 & 6548.00 & 6521.00 & 6464.32 & 6606.11 & 6172.56 & 6351.29 \\
\hline Hebi & 6538.60 & 6987.25 & 7080.00 & 6982.00 & 6897.00 & 6903.00 & 7005.00 & 7076.00 & 7007.03 & 7040.97 & 6830.27 & 6950.2 \\
\hline Xinxiang & 5810.60 & 6105.38 & 6158.00 & 6139.00 & 6083.00 & 6085.00 & 6338.00 & 6251.00 & 6228.04 & 6257.00 & 5481.13 & 5991.63 \\
\hline Jiaozuo & 7143.97 & 7434.48 & 7325.00 & 7307.00 & 7290.00 & 7270.00 & 7435.00 & 7409.00 & 7222.61 & 7240.23 & 6948.33 & 7128.25 \\
\hline Puyang & 6356.16 & 6684.94 & 6754.00 & 6601.00 & 6512.00 & 6437.00 & 6531.00 & 6346.00 & 6957.39 & 6592.94 & 6124.03 & 6395.79 \\
\hline Xuchang & 6235.63 & 6295.53 & 6689.00 & 6379.00 & 6450.00 & 6163.00 & 6271.00 & 6288.00 & 5891.07 & 6311.49 & 6218.68 & 6092.37 \\
\hline Luohe & 6429.01 & 6337.09 & 6185.00 & 5925.00 & 6037.00 & 6048.00 & 6450.00 & 6450.00 & 6308.65 & 6361.34 & 6495.89 & 6471.65 \\
\hline Sanmenxia & 4375.00 & 4704.17 & 4437.00 & 4384.00 & 4369.00 & 4280.00 & 4429.00 & 4334.00 & 4031.92 & 4180.53 & 4171.98 & 4060.14 \\
\hline Nanyang & 5295.35 & 5579.64 & 5569.00 & 5496.00 & 5468.00 & 5377.00 & 5551.00 & 5466.00 & 4924.29 & 5175.87 & 4910.57 & 4983.65 \\
\hline Shangqiu & 6206.25 & 6690.63 & 6693.00 & 6695.00 & 6613.00 & 6631.00 & 6664.00 & 6556.00 & 6351.80 & 6401.98 & 6150.62 & 6260.32 \\
\hline Xinyang & 5513.61 & 4943.29 & 4012.00 & 4310.00 & 4167.00 & 4384.00 & 4497.00 & 4494.00 & 4442.81 & 4476.29 & 6536.39 & 5362.3 \\
\hline Zhoukou & 6712.17 & 6119.65 & 5830.00 & 5451.00 & 5491.00 & 5954.00 & 6270.00 & 6081.00 & 5830.39 & 6241.08 & 5969.75 & 6110.87 \\
\hline Zhumadian & 5844.32 & 4955.39 & 4935.00 & 4971.00 & 5059.00 & 5225.00 & 5381.00 & 5399.00 & 5260.43 & 5441.36 & 5244.28 & 5482.4 \\
\hline Jiyuan & 4985.95 & 5344.99 & 5342.00 & 5233.00 & 5206.00 & 5219.00 & 5342.00 & 5356.00 & 5105.63 & 5198.11 & 5077.38 & 5127.7 \\
\hline
\end{tabular}

Table 1. Yield per unit area of maize in 18 cities of Henan Province in 2006-2017.

Unit: $\mathrm{kg} / \mathrm{ha}$. 
Table 2. Trends yield per unit area of maize in 18 cities of Henan Province in 2006-2017.

\begin{tabular}{|c|c|c|c|c|c|c|c|c|c|c|c|c|}
\hline & 2006 & 2007 & 2008 & 2009 & 2010 & 2011 & 2012 & 2013 & 2014 & 2015 & 2016 & 2017 \\
\hline Zhengzhou & 4969.87 & 4935.68 & 4901.20 & 4866.64 & 4831.88 & 4797.24 & 4762.99 & 4728.70 & 4694.49 & 4660.76 & 4626.62 & 4592.44 \\
\hline Luoyang & 5052.66 & 4986.95 & 4920.31 & 4854.07 & 4790.25 & 4731.76 & 4677.65 & 4626.22 & 4577.10 & 4531.21 & 4485.45 & 4439.35 \\
\hline $\begin{array}{l}\text { Pingding } \\
\text { shanNaNn }\end{array}$ & 4924.43 & 4880.11 & 4833.77 & 4783.77 & 4730.76 & 4674.99 & 4616.81 & 4556.69 & 4497.67 & 4445.59 & 4395.02 & 4344.09 \\
\hline Anyang & 6460.09 & 6473.92 & 6484.55 & 6490.03 & 6490.35 & 6486.26 & 6478.23 & 6465.67 & 6448.71 & 6428.01 & 6404.41 & 6380.51 \\
\hline Hebi & 6835.54 & 6868.02 & 6897.53 & 6922.29 & 6942.36 & 6958.37 & 6970.51 & 6978.43 & 6982.11 & 6982.50 & 6980.81 & 6978.84 \\
\hline Xinxiang & 6047.38 & 6071.29 & 6092.84 & 6109.99 & 6121.37 & 6125.89 & 6122.09 & 6108.09 & 6084.17 & 6052.04 & 6014.85 & 5977.79 \\
\hline Puyang & 6582.70 & 6581.14 & 6577.32 & 6570.00 & 6559.73 & 6547.36 & 6533.25 & 6516.69 & 6496.90 & 6471.44 & 6442.45 & 6413.27 \\
\hline Xuchang & 6406.48 & 6390.25 & 6372.30 & 6350.00 & 6323.83 & 6294.62 & 6264.42 & 6233.98 & 6204.10 & 6176.15 & 6148.35 & 6120.26 \\
\hline Luohe & 6214.08 & 6210.12 & 6208.31 & 6212.07 & 6224.58 & 6246.17 & 6275.26 & 6308.32 & 6343.55 & 6380.58 & 6418.67 & 6456.91 \\
\hline Sanmenxia & 4531.40 & 4495.15 & 4457.34 & 4418.49 & 4378.92 & 4338.62 & 4297.45 & 4254.73 & 4211.04 & 4167.80 & 4124.61 & 4081.20 \\
\hline Nanyang & 5551.14 & 5525.17 & 5496.63 & 5463.52 & 5424.56 & 5378.76 & 5325.62 & 5264.56 & 5197.31 & 5127.59 & 5056.37 & 4985.14 \\
\hline Shangqiu & 6583.45 & 6589.26 & 6591.29 & 6586.80 & 6574.03 & 6552.34 & 6521.44 & 6481.87 & 6435.55 & 6385.18 & 6332.59 & 6279.81 \\
\hline Xinyang & 4657.31 & 4602.79 & 4556.84 & 4531.42 & 4533.05 & 4566.04 & 4631.03 & 4726.84 & 4850.96 & 4998.55 & 5160.67 & 5323.19 \\
\hline Zhoukou & 6120.11 & 6063.61 & 6013.03 & 5974.86 & 5953.73 & 5949.07 & 5955.66 & 5968.34 & 5985.07 & 6004.97 & 6025.59 & 6046.85 \\
\hline Jiyuan & 5216.68 & 5226.61 & 5234.24 & 5238.43 & 5239.15 & 5236.29 & 5229.43 & 5217.95 & 5202.38 & 5184.61 & 5165.60 & 5146.39 \\
\hline
\end{tabular}

Unit: kg/ha.

The state transition probability matrix is

$$
P_{1}^{(1)}=\left[\begin{array}{ll}
\frac{1}{3} & \frac{2}{3} \\
\frac{4}{5} & \frac{1}{5}
\end{array}\right]
$$

As Zhengzhou maize is in the state of reduced production in 2017, according to the maximum probability criterion, it can be obtained

$$
\max \left\{p_{11}^{(1)}, p_{12}^{(1)}\right\}=p_{12}^{(1)}=\frac{2}{3}
$$

Therefore, it can be found that Zhengzhou 2018 maize will increase production.

The same principle is available that the state transition probability matrix of Kaifeng City, Luoyang City, Pingdingshan City, Anyang City, Hebi City, Xinxiang City, Jiaozuo City, Fuyang City, Xuchang City, Luohe City, Sanmenxia City, Nanyang City, Shangqiu City, Xinyang City, Zhoukou City, Zhumadian City and Jiyuan City are as follows: 


$$
\begin{aligned}
& P_{2}^{(1)}=\left[\begin{array}{ll}
\frac{3}{7} & \frac{4}{7} \\
1 & 0
\end{array}\right], P_{3}^{(1)}=\left[\begin{array}{ll}
\frac{1}{4} & \frac{3}{4} \\
\frac{3}{7} & \frac{4}{7}
\end{array}\right], P_{4}^{(1)}=\left[\begin{array}{cc}
0 & \frac{1}{5} \\
\frac{3}{8} & \frac{5}{8}
\end{array}\right], P_{5}^{(1)}=\left[\begin{array}{cc}
\frac{1}{2} & \frac{1}{2} \\
\frac{2}{7} & \frac{5}{7}
\end{array}\right], P_{6}^{(1)}=\left[\begin{array}{cc}
\frac{1}{2} & \frac{1}{2} \\
\frac{2}{7} & \frac{5}{7}
\end{array}\right], \\
& P_{7}^{(1)}=\left[\begin{array}{ll}
\frac{1}{4} & \frac{3}{4} \\
\frac{2}{7} & \frac{5}{7}
\end{array}\right], P_{8}^{(1)}=\left[\begin{array}{ll}
\frac{1}{2} & \frac{1}{2} \\
\frac{3}{5} & \frac{2}{5}
\end{array}\right], P_{9}^{(1)}=\left[\begin{array}{ll}
\frac{2}{3} & \frac{1}{3} \\
\frac{2}{5} & \frac{3}{5}
\end{array}\right], P_{10}^{(1)}=\left[\begin{array}{ll}
\frac{1}{4} & \frac{3}{4} \\
\frac{3}{7} & \frac{4}{7}
\end{array}\right], P_{11}^{(1)}=\left[\begin{array}{cc}
\frac{2}{3} & \frac{1}{3} \\
\frac{2}{5} & \frac{3}{5}
\end{array}\right], \\
& P_{12}^{(1)}=\left[\begin{array}{ll}
\frac{1}{2} & \frac{1}{2} \\
\frac{3}{5} & \frac{2}{5}
\end{array}\right], P_{13}^{(1)}=\left[\begin{array}{ll}
\frac{1}{4} & \frac{3}{4} \\
\frac{3}{7} & \frac{4}{7}
\end{array}\right] P_{14}^{(1)}=\left[\begin{array}{ll}
\frac{1}{3} & \frac{2}{3} \\
\frac{1}{4} & \frac{3}{4}
\end{array}\right], P_{15}^{(1)}=\left[\begin{array}{ll}
\frac{7}{8} & \frac{1}{8} \\
\frac{1}{3} & \frac{2}{3}
\end{array}\right], P_{16}^{(1)}=\left[\begin{array}{cc}
\frac{2}{5} & \frac{3}{5} \\
\frac{1}{2} & \frac{1}{2}
\end{array}\right], \\
& P_{17}^{(1)}=\left[\begin{array}{ll}
\frac{1}{2} & \frac{1}{2} \\
\frac{3}{5} & \frac{2}{5}
\end{array}\right], P_{18}^{(1)}=\left[\begin{array}{ll}
\frac{1}{2} & \frac{1}{2} \\
\frac{3}{5} & \frac{2}{5}
\end{array}\right],
\end{aligned}
$$

\section{Results Analysis}

Thus, Kaifeng, Luoyang, Pingdingshan, Xinxiang, Jiaozuo, Xuchang, Luohe, Nanyang, Shangqiu and Xinyang may increase their maize production in 2018, while Puyang and Zhumadian will reduce production. Anyang, Hebi, Jiaozuo, Sanmenxia, Zhoukou, Jiyuan, these areas maize production increase or decrease situation can not be determined.

The geographical location of 18 cities in Henan Province is shown in Figure 1. By observing the map and combining the results of Markov forecast, the following conclusions can be obtained: Around Anyang are Xinxiang, Puyang, Hebi, Anyang including Hebi. According to Markov forecast, Xinxiang will increase production in 2018, while Puyang will reduce production and Hebi is uncertain, but Anyang obviously has more border areas with Xinxiang. As a result, Anyang may increase its maize production in 2018. Hebi is surrounded by Anyang and Xinxiang, Anyang and Xinxiang are both increasing production, so it is predicted that Hebi' maize will also increase production in 2018. Jiaozuo is surrounded by Xinxiang, Luoyang, Zhengzhou and Jiyuan, and mainly Xinxiang, Luoyang and Zhengzhou. In these three cities, maize in 2018 is predicted to increase production, so it is predicted that Jiaozuo maize may increase production. Around Jiyuan is Luoyang and Jiaozuo. As the two cities' maize production predictions may increase production, it is predicted that Jiyuan' maize may increase production in 2018. Around Sanmenxia, it is Luoyang and Nanyang. Both cities have increased production. Therefore, it is estimated that Sanmenxia' maize may increase production in 2018. Around Zhoukou, there are Shangqiu, Kaifeng, Xuchang, Luohe and Zhumadian. Except Zhumadian, all other cities are expected to increase production. From the perspective of contact area, it is predicted that Zhoukou's 2018 maize production may also increase production. 


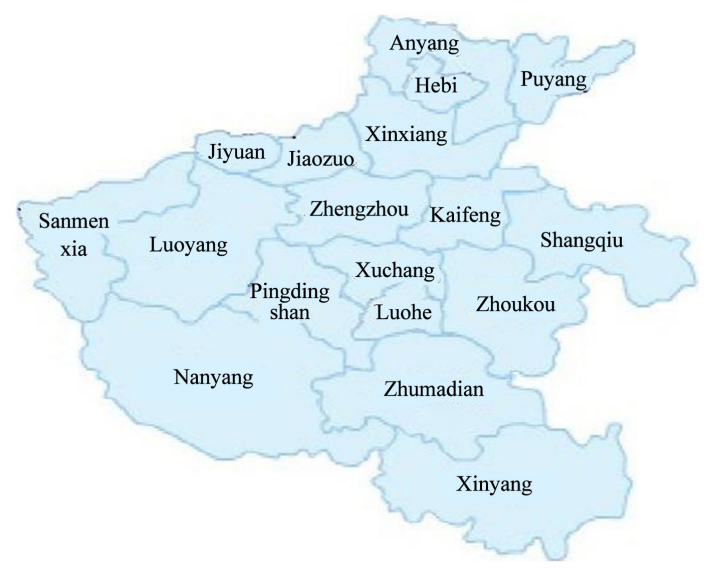

Figure 1. Geographic map of 18 cities in Henan Province.

According to the forecast, it is found that most areas of Henan Province increased production in 2018, which is more in line with the actual situation. Although the meteorological anomalies will make maize yield decrease to a certain extent, with the improvement of scientific and technological level, People take precautions against meteorological anomalies in advance and compensate the yield losses caused by meteorological anomalies to a certain extent, thus increasing maize production in Henan Province on the whole.

\section{Policy Recommendations}

Through improving the varieties of maize, selecting and cultivating new varieties with strong resistance, and adjusting the production structure of grain, it can improve its ability to defend against meteorological anomalies, reduce the losses caused by agricultural meteorological anomalies, and ensure stable production and increase of grain production.

We should vigorously strengthen the construction of farmland and water conservancy and do a good job in the construction of agricultural infrastructure projects such as well irrigation and water-saving irrigation. It is necessary to promote the comprehensive utilization of straw returning to the field, protect the ecological environment, avoid over-exploitation and utilization of land resources, and improve the quality of farmland.

Strengthen research on the prevention of agrometeorological disasters. It is necessary to increase the systematic analysis of disasters and explore its complex background to develop detailed supporting defense measures. Through the research on the disaster early warning system, the ability to predict and forecast disasters will be improved. Conduct in-depth research on meteorological anomalies and other disciplines to improve the response of maize to meteorological anomalies and reduce the impact of meteorological anomalies on maize yield.

\section{Conclusion}

In order to improve grain production and ensure national grain security, considering the influence of meteorological factors on grain production, the grain 
production reduction in Henan Province was predicted in order to prevent the grain production in advance. GM $(1,1)$ model is used to predict the yield of maize in 2017 in Henan Province, the trend yield of maize is obtained by using HP filter method, and then the meteorological yield is obtained. Finally, Markov model is used to predict the yield of maize in 18 cities of Henan Province in 2018. The results showed that maize production in most cities of Henan Province is likely to increase in 2018, and only a small part of the maize may reduce production. This has a great relationship with the improvement of science and technology and agricultural production facilities in Henan Province. Strengthening the prediction of meteorological anomalies and doing a good job of prevention will have a great impact on ensuring stable production and increasing yield of maize in Henan Province.

\section{Acknowledgements}

The authors are grateful to anonymous referees for their helpful and constructive comments on this paper. The work was supported by the Soft-science Foundation of Henan Province (172400410015), and the Philosophy and Social Program of Henan Province (2016BJJ022).

\section{Conflicts of Interest}

The authors declare no conflicts of interest regarding the publication of this paper.

\section{References}

[1] Henan Provincial Bureau of Statistics (2017) Henan Statistical Yearbook. China Publishing House, Beijing.

[2] Ma, S.Q., Wang, Q., Chen, F.T., et al. (2015) Impact of Spring Maize Seeding Growth on Yield and Assessment Models of Production Cut under Background of Spring Drought. Transactions of the Chinese Society of Agricultural Engineering, 31, 171-179.

[3] Sun, Y.L., Bian, X.J., Huang, C.X., et al. (2013) Climate Prediction of Corn Regionalization and Yield in Frigid and Arid Area in Linxia, Gansu. Journal of Northwest $A \& F$ University (Natural Science Edition), 41, 49-55.

[4] Luo, Z.Y. and Tang, Y. (2013) The Prediction Analysis on Maize Yield Changes in Heilonjiang Province-Empirical Research Based on Support Vector Machine (SVM). Journal of Agricultural Mechanization Research, 2, 30-34.

[5] Zhang, W.Y., Li, Q.S. and Li, Y.Q. (2007) Analysis and Forecast of Corn Yield of Hebei Province with Grey System. Acta Agriculturae Boreali-Sinica, 22, 160-162.

[6] Li, G.Q., Lu, X.F., Cao, Z.Y., et al. (2017) Analysis and Grey Model Prediction of Maize Yield in Huanghuaihai Region from 2001 to 2015. Bulletin of Agricultural Science and Technology, 9, 85-87.

[7] Jiang, H.W., Zhao, M., Guo, T.T., et al. (2011) Grey Prediction of Grain Yield Based on Period Residual Modification Method in Liaoning Province. Journal of Meteorology and Environment, 27, 51-55.

[8] Fang, H.M., Zhang, N.T., Ding, W.K., et al. (2007) Study on Forecasting the Yield in 
Maize Regional Test Based on BP Neural Network. Journal of Anhui Agricultural Sciences, 35, 10969-10970.

[9] Su, H.Q., Chen, G.F. and Zhu, C.R. (2010) A Combination Forecasting Model for Corn Yield Based on Entropy Weight. Journal of Shenyang Agricultural University, 41, 125-127.

[10] Hou, Y.Y., Wang, J.L., Mao, L.X., Song, Y.B., et al. (2009) Dynamic Estimation Models of Corn and Wheat Yields in USA Based on Remote Sensing Data. Chinese Journal of Ecology, 28, 2142-2146.

[11] Wei, J., Ning, J. and Li, F.Z. (2015) Forecasting of Corn Production in Shanxi Province-Based on the Exponential Smoothing Model. Tianjin Agricultural Sciences, 21, 84-85+97.

[12] Ma, J.R., Tian, J.C., Shen, H. and Sun, F. (2018) The Model of Water and Fertilizer Coupling of Maize Drip Irrigation Under Plastic Films and Research on Yield Effect in Pumping Irrigation Areas. China Rural Water and Hydropower, 4, 10-20.

[13] Wang, F.T. (1993) Impacts of $\mathrm{CO}_{2}$ Concentration Increase in Atmosphere on Plant Growth and Agricultural Production. Meteorological Monthly, 19, 8-13.

[14] Zhang, J.P., Zhao, Y.X., Wang, C.Y., et al. (2008) Simulation of Maize Production under Climate Change Scenario in Northeast China. Chinese Journal of Eco-Agriculture, 16, 1448-1452. https://doi.org/10.3724/SP.J.1011.2008.01448

[15] Jin, Z.Q., Ge, D.K., Zheng, X.L. and Chen, H. (1996) Assessing the Potential Impacts of Global Climate Change on Maize Production in China. Acta Agronomica Sinica, 22, 513-524.

[16] Xiong, W., Feng, Y.Z., Gao, Q.Z. and Li, Y.C. (2011) Impacts of Climate Change on Irrigated Maize Production in Shiyang and Daling River Basins. Arid Land Geography, 34, 150-159.

[17] Chen, S., Dou, Z.H., Jiang, T.C., Li, H.L., et al. (2017) Maize Yield Forecast with DSSAT-CERES-Maize Model Driven by Historical Meteorological Data of Analogue Years by Clustering Algorithm. Transactions of the Chinese Society of Agricultural Engineering, 33, 147-155.

[18] Shang, Z.B. (2000) The Potential Impacts of Global Climate Change on Spring-Maize Growth in Shenyang. Chinese Bulletin of Botany, 42, 300-305.

[19] Ji, Q. (2012) Prediction of Maize Yield Based on Logistic and Disaster Decrement Rate Method. Chinese Agricultural Science Bulletin, 28, 293-296.

[20] Deng, J.L. (1982) Control Problem of Grey System. System \& Control Letter, 1, 288-294. https://doi.org/10.1016/S0167-6911(82)80025-X

[21] Deng, J.L. (1988) Grey Forecasting and Decision Making. Huazhong University of Science and Technology Press, Wuhan.

[22] Liu, Y.K., Xie, C.L., Yu, Z.J. and Ling, S.H. (2011) Trend Prediction Methods Study of Nuclear Power Plant Based on GM $(1,1)$ and Grey Markov GM $(1,1)$ Models. Atomic Energy Science and Technology, 45, 1075-1079.

[23] Zhang, Q.Y., Zhou, X.H. and Wang, W.T. (2007) The Forecast for the Wear Trend of the Diesel Engine Based on Grey Markov Chain Model. Lubrication Engineering, 9, 145-147.

[24] Tang, D.D. (2007) Three Frequency Selective Filters and Their Applications in China. The Journal of Quantitative \& Technical Economics, 9, 144-156.

[25] Fang, S.B. (2011) Exploration of Method for Discrimination between Trend Crop Yield and Climatic Fluctuant Yield. Journal of Natural Disasters, 20, 13-18.

[26] Wang, Y. and Fang, X.Q. (2004) A Method for Calculating the Climatic Yield of Grain under Climate Change. Journal of Natural Resources, 19, 531-536. 